\title{
DigitalMicrograph Script Source Listing for a Geometric Phase Analysis
}

\author{
Kyou-Hyun Kim* \\ Advanced Process and Materials R\&BD Group, Incheon Regional Division, \\ Korea Institute of Industrial Technology, Incheon 406-840, Korea
}

*Correspondence to: $\mathrm{Kim} \mathrm{KH}$,

Tel: +82-32-850-0431

Fax: $+82-32-850-0430$

E-mail: khkim1308@kitech.re.kr

Received April 17, 2015

Revised June 25, 2015

Accepted June 25, 2015
Numerous digital image analysis techniques have been developed with regard to transmission electron microscopy (TEM) with the help of programming. DigitalMicrograph (DM, Gatan Inc., USA), which is installed on most TEMs as operational software, includes a script language to develop customized software for image analysis. Based on the DM script language, this work provides a script source listing for quantitative strain measurements based on a geometric phase analysis.

Key Words: DigitalMicrograph script, Transmission electron microscopy, Quantitative strain measurement, Geometric phase analysis

\section{INTRODUCTION}

Transmission electron microscopy (TEM) is a powerful tool for analyzing a broad range of materials with a variety of analysis techniques. Conventional TEM analysis techniques have been improved continuously since TEM was developed in 1930s. One recent advance is the incorporation of aberration correctors, which drastically enhances the resolution of TEM. On the other hand, numerous data analysis techniques based on post-image processing have also been introduced in an effort to obtain more information from raw TEM data, which is scarcely distinguishable by empirical methods or manual calculations (Buseck et al., 1988; Reetz et al., 2000; Zuo et al., 2003; Huang et al., 2009; Kim et al., 2013a).

Several tools, such as Matlab, $\mathrm{C} / \mathrm{C}++$ and Python, can be used to develop software for advanced image processing from TEM images. DigitalMicrograph (DM, Gatan Inc., USA) also provides a scripting language called DM script for developing data analysis software for TEM. The syntax of DM is very similar to that of $\mathrm{C} / \mathrm{C}++$. Unlike other programming languages, however, DM script simultaneously serves as a compiler to create an object file, as a linker to create an executable file, and as a runtime environment. More precisely,
DM works as not a low-level language but as an interpreter for customized scripts. For this reason, its computation speed is relatively slow. Nevertheless, DM script provides a convenient programming environment with a variety of functions to manipulate images and to access the TEM instrument for customized operations. Several analysis techniques have already been reported based on the DM script (Kim \& Zuo, 2013; Kim et al., 2015). Kim and colleagues recently introduced new algorithms to quantify the symmetry recorded in convergent beam electron diffraction (CBED) patterns and to perform scanning CBED for symmetry mapping (Kim et al., 2012; Kim, 2013; Kim et al., 2013b; Kim \& Zuo, 2013). Also, the DM script database contains a considerable number of applications for customized software scripting using the DM script language (FELMI-ZFE [Internet]).

Using the advantages of DM script, this work provides a DM script source listing for quantitative strain measurements. Quantitative strain, or displacement measurement, is a widely used image processing technique for materials. Algorithms for strain mapping are mainly classified into two groups in terms of real and reciprocal space. The Peak Pairs algorithm (PPA) calculates the local displacement using the intensity maximum of the peaks between each pair of neighboring lattice points

This work was supported by a research grant from the Korea Institute of Industrial Technology (KITECH).

@ This is an open-access article distributed under the terms of the Creative Commons Attribution Non-Commercial License (http://creativecommons.org/licenses/by-nc/4.0) which permits unrestricted noncommercial use, distribution, and reproduction in any medium, provided the original work is properly cited.

Copyrights @ 2015 by Korean Society of Microscopy 
in real space (Galindo et al., 2007). Another algorithm known as the geometric phase analysis was proposed by Hÿtch et al. (1998). Geometric phase analysis (GPA) is based on phase retrieval calculated in the Fourier (reciprocal) space. While the two techniques are complementary to each other, PPA can fail to calculate the local displacement with a complex lattice structure (Galindo et al., 2007). In addition, GPA is created relatively easily with DM script language considering the computation process of the DM script. As mentioned earlier, DM script only works as an interpreter, unlike other low-level programming languages. It therefore requires a significant amount of computation time for a complex loop structure. The PPA algorithm requires a pixel-by-pixel operation to find neighboring lattice points such that the computation time increases rapidly, even for a small two-dimensional image. A dynamic-link library (dll) file may need to be created using $\mathrm{C}++$ in order to create the script for PPA on DM. In contrast, the DM script has the basic functions for basic Fourier/ inverse Fourier transform and image processing, which are the major functions for the GPA algorithm. This results in a reasonable computation time reduction on DM. In this report, a DM script source listing for GPA is created and listed in the Appendix (online only at http://www.appmicro.org) to be used directly on DM.

\section{THEORY}

Hÿtch et al. $(1997,1998)$ proposed a means of quantifying local displacement and strain fields from a high-resolution (HR) image recorded using TEM. This section will briefly introduce the fundamental theory of GPA as proposed by Hÿtch et al. $(1997,1998)$. More details about the GPA can be found in the literature (Hÿtch et al., 1998).

The image intensity at position $r$ can be expressed as follows,

$$
\mathrm{I}(\boldsymbol{r})=\sum_{g} \mathrm{H}_{g}(\boldsymbol{r}) \exp \{2 \pi \mathrm{ig} \cdot \boldsymbol{r}\},
$$

where $\boldsymbol{g}$ is the Bragg position and $\boldsymbol{r}$ is the position vector of intensity at a pixel $(\mathrm{x}, \mathrm{y})$ in an image. The Fourier coefficient, $\mathrm{H}_{g}(\boldsymbol{r})$, is then described as

$$
\mathrm{H}_{g}(\boldsymbol{r})=\mathrm{A}_{g}(\boldsymbol{r}) \exp \left\{\mathrm{iP}_{g}(\boldsymbol{r})\right\}
$$

where the amplitude $\mathrm{A}_{g}(\boldsymbol{r})$ and phase $\mathrm{P}_{g}(\boldsymbol{r})$ respectively represent the contrast level and lateral position of the fringes in the raw image. The inverse Fourier transform of equation

(2) results in the complex image $\mathrm{H}_{g}^{\prime}(\boldsymbol{r})$ :

$$
\mathrm{H}_{g}^{\prime}(\boldsymbol{r})=\mathrm{H}_{g}(\boldsymbol{r})\{2 \pi \mathrm{ig} \cdot \boldsymbol{r}\}
$$

Equation 2 is substituted into equation (3) for $\mathrm{H}_{g}(\boldsymbol{r})$.

$$
\mathrm{H}_{g}^{\prime}(\boldsymbol{r})=\mathrm{A}_{g}(\boldsymbol{r})\left\{2 \pi \mathrm{ig} \cdot \boldsymbol{r}+\mathrm{iP}_{g}(\boldsymbol{r})\right\}
$$

Equation (4) is then used to calculate the Bragg-filtered image intensity, amplitude, phase, and raw-phase images from the original image in the following manner,

Bragg-filtered image intensity: $\mathrm{B}_{g}(\boldsymbol{r})=2 \mathrm{Real}\left[\mathrm{H}_{g}^{\prime}(\boldsymbol{r})\right]$

Amplitude image: $\mathrm{A}_{g}(\boldsymbol{r})=\operatorname{Mod}\left[\mathrm{H}_{g}^{\prime}(\boldsymbol{r})\right]$

Phase image: $\mathrm{P}_{g}(\boldsymbol{r})=$ Phase $\left[\mathrm{H}_{g}^{\prime}(\boldsymbol{r})\right]-2 \pi \boldsymbol{g} \cdot \boldsymbol{r}$

$$
\text { Raw-phase image: } \mathrm{P}_{g}(\boldsymbol{r})=\text { Phase }\left[\mathrm{H}_{g}^{\prime}(\boldsymbol{r})\right] \text {, }
$$

where Real, Mod and Phase, respectively denote the real part, modulus and phase of $\left[\mathrm{H}_{g}^{\prime}(\boldsymbol{r})\right]$. The two-dimensional displacement, $\mathrm{u}(\boldsymbol{r})$, is then calculated from equation (7) as follows:

$$
\mathrm{u}(\boldsymbol{r})=-\frac{1}{2 \pi}\left[\mathrm{P}_{g_{1}}(r) a_{1}+\mathrm{P}_{g_{2}}(r) a_{2}\right.
$$

In this equation, the vectors $\boldsymbol{a}_{1}$ and $\boldsymbol{a}_{2}$ correspond to the lattice vectors in real space as defined by the reciprocal lattice vectors of $\boldsymbol{g}_{1}$ and $\boldsymbol{g}_{2}\left(\boldsymbol{g}_{i} \cdot \boldsymbol{a}_{j}=\boldsymbol{\delta}_{i j}\right)$. The strain and rotation tensors are finally given by the derivatives of the two-dimensional displacement, $\mathrm{u}(\boldsymbol{r})$,

$$
\begin{aligned}
& =\varepsilon=\frac{1}{2}\left\{e+e^{T}\right\} \\
& =\omega=\frac{1}{2}\left\{e-e^{T}\right\}
\end{aligned}
$$

where $e=\left(\begin{array}{ll}e_{x x} & e_{x y} \\ e_{y x} & e_{y y}\end{array}\right)=\left(\begin{array}{ll}\frac{\partial u_{x}}{\partial x} & \frac{\partial u_{x}}{\partial y} \\ \frac{\partial u_{y}}{\partial x} & \frac{\partial u_{y}}{\partial y}\end{array}\right)=-\frac{1}{2 \pi}\left(\begin{array}{cc}a_{1 x} & a_{2 x} \\ a_{1 y} & a_{2 y}\end{array}\right)\left(\begin{array}{ll}\frac{\partial P_{g 1}}{\partial x} & \frac{\partial P_{g 1}}{\partial y} \\ \frac{\partial P_{g 2}}{\partial x} & \frac{\partial P_{g 2}}{\partial y}\end{array}\right)$.

\section{THE PROGRAM}

Fig. 1 shows the GPA software installed on DM. In the Appendix, the script source listing can be found and can simply be installed on DM included in Gatan Microscopy Suite (GMS, version 2.11, 32-bit architecture; Gatan Inc.). GMS can be freely downloaded from Gatan's website.

A fast Fourier transform or power spectrum image is initially calculated from an HR image in order to measure the local strain, rotation and displacement. For the power spectrum image, the software provides a "Gaussian edge smoothing" function to make spots more distinguishable in the calculated power spectrum. From the power spectrum, any two nonlinear $\boldsymbol{g}$-vectors can be chosen using two virtual apertures (the oval annotations in DM). Fig. 2 shows an example of

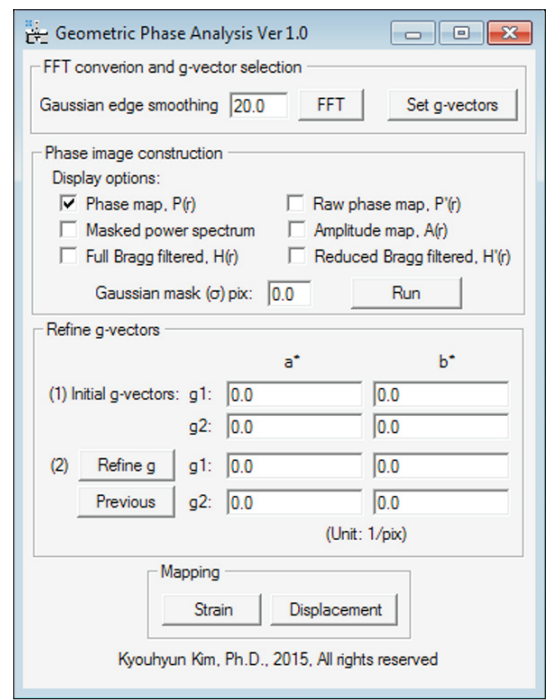

Fig. 1. Dialogue box for the geometric phase analysis software installed on DigitalMicrograph (Gatan Inc., USA). 

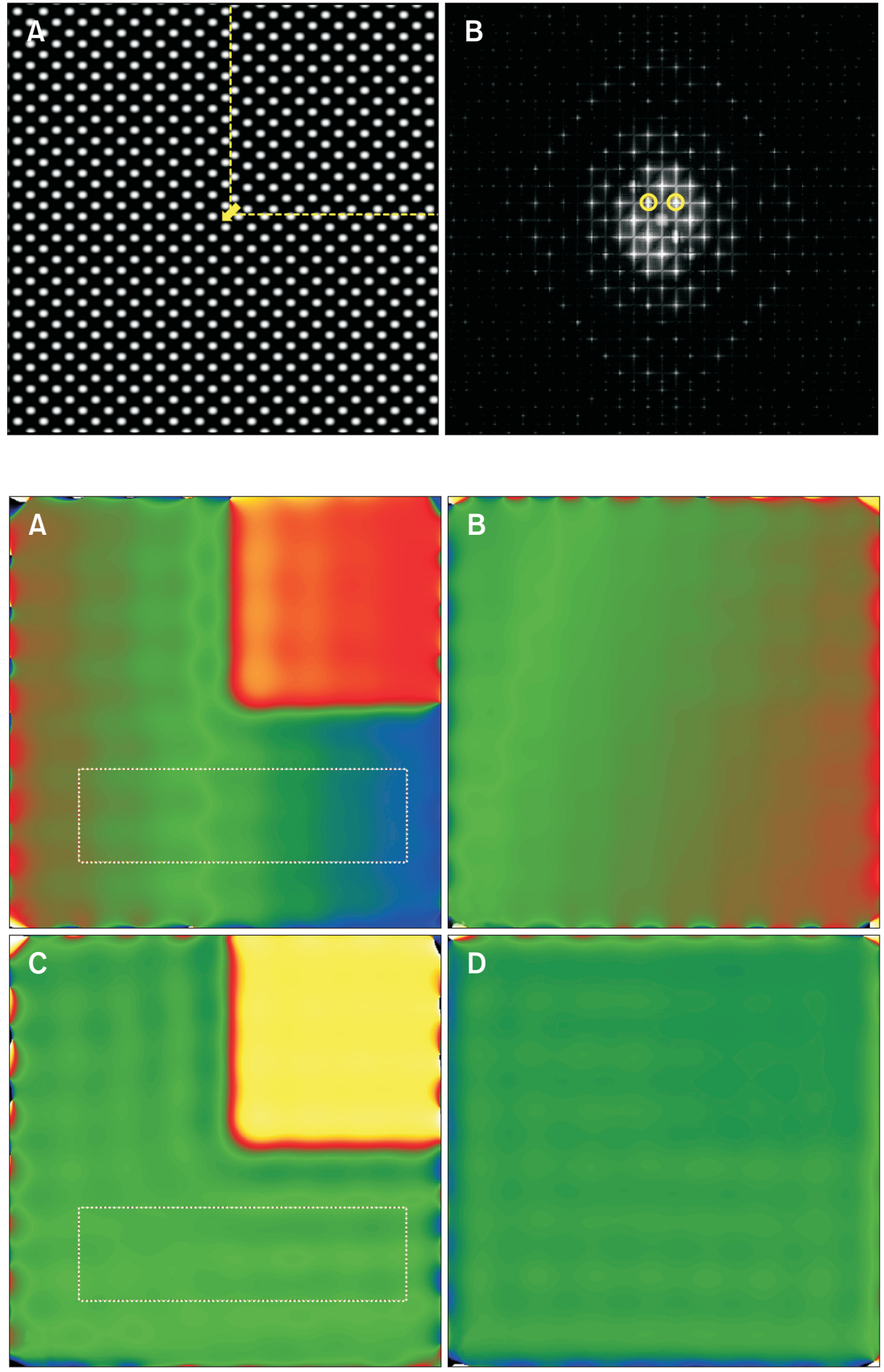

\section{D}

a simulated HR image and Fig. 2B is the power spectrum calculated from Fig. 2A with a Gaussian edge smoothing value of 50. In order to determine two $g$-vectors, two non-linear spots are selected with the oval annotations (yellow circles) as shown in Fig. 2B and are then stored in the software using the "Set $g$-vectors" function. In this process, two reciprocal lattice
Fig. 2. (A) Simulated high-resolution image for geometric phase analysis. (B) Gaussian-edge-smoothed power spectrum calculated from Fig. 2A.
Fig. 3. (A, B) Calculated $\mathrm{P}(r)$ image for two $g$-vectors. (C, D) $\mathrm{P}(r)$ images with the refined $g$-vectors. A region-of-interest box automatically appears on one of the $\mathrm{P}(r)$ images to refine the selected $g$-vectors. vectors are determined by the distance from the center of the power spectrum to the intensity maximum of the peaks. The intensity maximum can be calculated with sub-pixel accuracy based on the center of mass of the intensity of the peaks (the CenterOfMass subroutine in the script source; please see the appendix). 

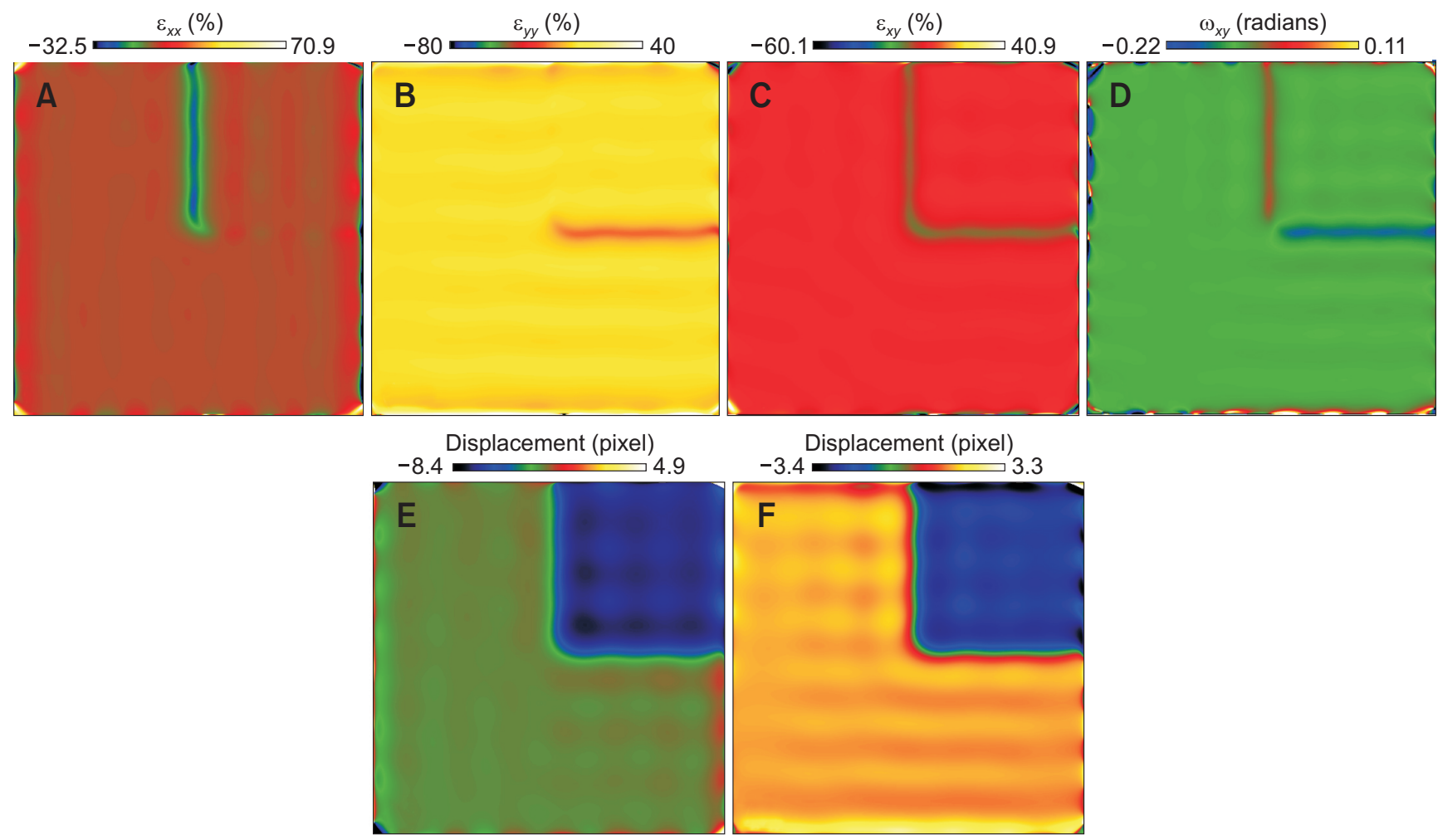

Fig. 4. Calculated strain maps for $\varepsilon_{\mathrm{xx}}(\mathrm{A}), \varepsilon_{\mathrm{yy}}(\mathrm{B})$, and $\varepsilon_{\mathrm{xy}}(\mathrm{C})$; rotation map for $\omega_{\mathrm{xy}}(\mathrm{D})$; two dimensional-displacement $\mathrm{u}(r)$ along $\mathrm{x}(\mathrm{E})$ and $\mathrm{y}(\mathrm{F})$.

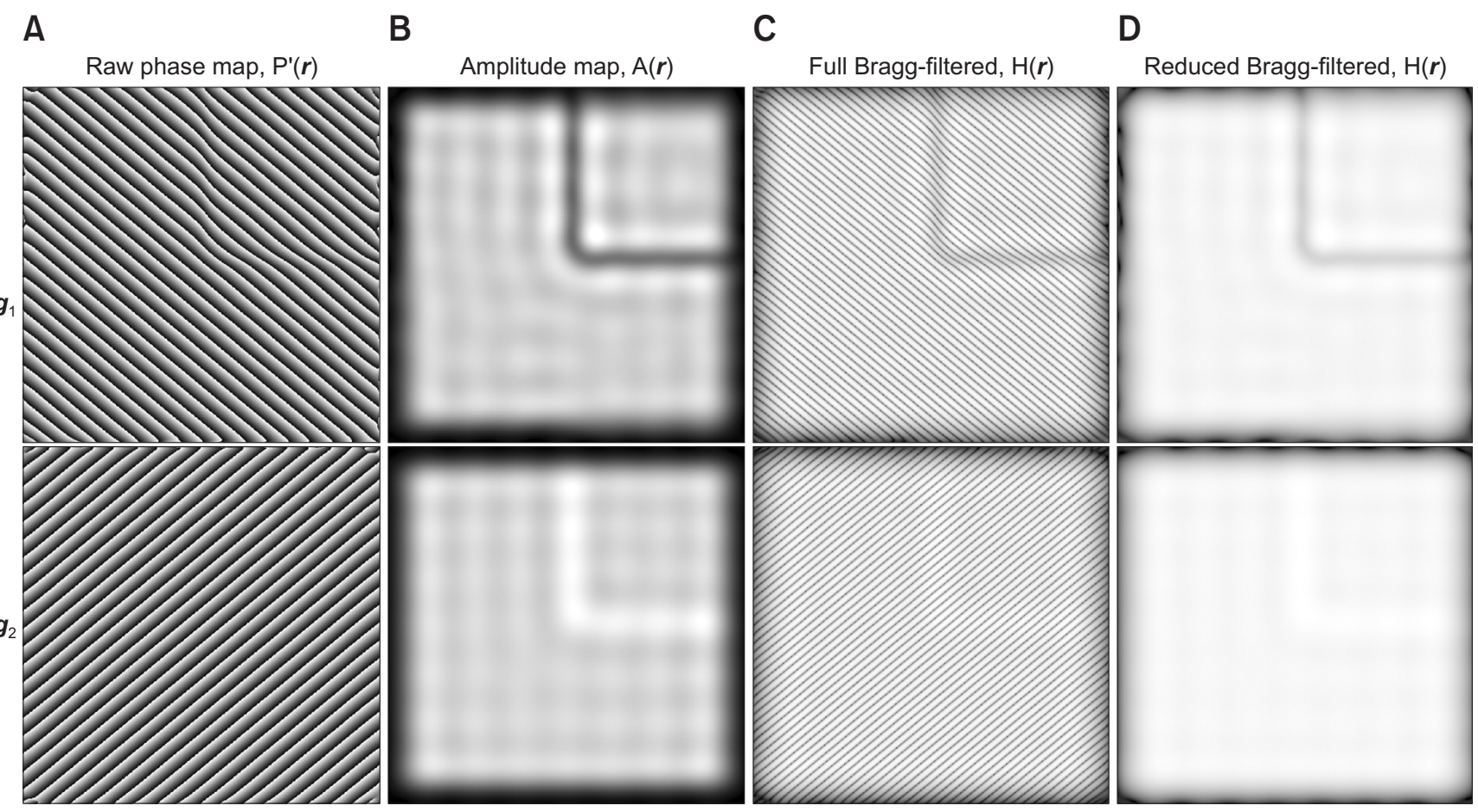

Fig. 5. Raw phase map $\mathrm{P}^{\prime}(\boldsymbol{r})(\mathrm{A})$, amplitude map $\mathrm{A}(\boldsymbol{r})$ (B), full Bragg-filtered map $\mathrm{H}(\boldsymbol{r})(\mathrm{C})$, and reduced Bragg-filtered $\mathrm{H}(\boldsymbol{r})$ (D) for the lattice reciprocal vectors of $g_{1}$ and $g_{2}$. 
Once two $\boldsymbol{g}$-vectors are selected, a phase map, $\mathrm{P}(\boldsymbol{r})$, can be generated with the "Run" button in the phase image construction menu. Fig. 3 shows the generated $\mathrm{P}(\boldsymbol{r})$ images for the selected $\boldsymbol{g}$-vectors. From the first calculated $\mathrm{P}(\boldsymbol{r})$ images, the selected $\boldsymbol{g}$-vectors can be refined. As shown in Fig. 3A, a region-of-interest tool automatically appears on one of the $\mathrm{P}(\boldsymbol{r})$ images. The user then needs to choose a proper reference area and then refine the $g$-vectors using the "Refine-g" button. New $\mathrm{P}(\boldsymbol{r})$ images are then reloaded with the refined $g$-vectors using the "Run" button in the phase image construction menu. These procedures should be repeated until the $g$-vectors have the same values. Fig. 3C and D show the final $\mathrm{P}(\boldsymbol{r})$ images after several refinement procedures.

Based on the refined $\mathrm{P}(\boldsymbol{r})$ images, the local strain $(\varepsilon)$, rotation $(\omega)$ can be calculated using the "strain" button in the mapping menu (see Fig. 1). Fig. 4A-D show the calculated strain maps of $\varepsilon_{\mathrm{x}}$ (Fig. 4A), $\varepsilon_{\mathrm{y}}$ (Fig. 4B), $\varepsilon_{\mathrm{xy}}$ (Fig. 4C) and the rotation matrix $\omega_{x y}$ (Fig. 4D). The local strain fields are observed in the calculated strain maps. A user can also obtain the map of $u(\boldsymbol{r})$ by using the "displacement" button in the mapping menu. The two-dimensional displacements, $u(\boldsymbol{r})$, are used to calculate the strain maps with equation (9) to (11). Fig. $4 \mathrm{E}$ and $\mathrm{F}$ display $u(\boldsymbol{r})$ for the $\mathrm{x}$ and $\mathrm{y}$ direction, respectively.
The unit of $u(r)$ is determined by the unit of used images. Thus, Fig. 4E and $\mathrm{F}$ are represented based on the unit of pixel because the lattice vectors calculated from the simulated HR image have the unit of pixel.

Other images of the raw phase image, masked power spectrum, amplitude map, and full/reduced Bragg-filtered images can be also obtained from the software as shown in Fig. 5. From the raw phase image, the local reciprocal lattice $\boldsymbol{g}$-vectors are directly calculated by differentiation: $\nabla \mathrm{P}_{g}^{\prime}(\boldsymbol{r})=2 \pi \boldsymbol{g}(\boldsymbol{r})$. This is then used to calculate the phase image $\mathrm{P}_{\mathrm{g}}(\boldsymbol{r})$ by subtracting $2 \pi \mathrm{ig} \cdot \boldsymbol{r}$. The corresponding algorithm can be found in the Appendix (please see the "//Phase image, $\mathrm{P}(\mathrm{r})$ "). While the amplitude map and full/reduced Bragg-filtered images are not directly related to the calculation of strain map, they can be applied to image an antiphase domain boundary (Hÿtch, 1997) or dislocations (Hÿtch et al., 2003). In order to obtain the images shown in Fig. 5, a user needs to check "display options" in the phase image construction menu.

\section{CONFLICT OF INTEREST}

No potential conflict of interest relevant to this article was reported.

\section{REFERENCES}

Buseck P R, Epelboin Y, and Rimsky A (1988) Signal processing of highresolution transmission electron microscope images using Fourier transforms. Acta Cryst. A 44, 975-986.

FELMI-ZFE: DigitalMicrograph ${ }^{\mathrm{TM}}$ script database [Internet]. Available from: http://portal.tugraz.at/portal/page/portal/felmi/DM-Script.

Galindo P L, Kret S, Sanchez A M, Laval J, Yáñez A, Pizarro J, Guerrero E, Ben T, and Molina S I (2007) The peak pairs algorithm for strain mapping from HRTEM images. Ultramicroscopy 107, 1186-1193.

Hÿtch M J (1997) Geometric phase analysis of high resolution electron microscope images. Scanning Microscopy 11, 53-66.

Hÿtch M J, Pataux J L, and Penisson J M (2003) Measurement of the displacement field of dislocations to $0.03 \AA$ by electron microscopy. Nature 423, 270-273.

Hÿtch M J, Snoeck E, and Kilaas R (1998) Quantitative measurement of displacement and strain fields from HREM micrographs. Ultramicroscopy 74, 131-146.

Huang W J, Zuo J M, Jiang B, Kwon K W, and Shim M S (2009) Subangstrom-resolution diffractive imaging of single nanocrystals. Nature Phys. 5, 129-133.

Kim H, Meng Y, Rouviére J L, Isheim D, Seidman D N, and Zuo J M. (2013a) Atomic resolution mapping of interfacial intermixing and segregation in InAs/GaSb superlattices: a correlative study. J. Appl. Phys. 113, 103511.
Kim K H (2013) Local symmetry and polarization in relaxor-based ferroelectric crystals, Materials Science and Engineering, (University of Illinois at Urbana-Champaign, Champaign).

Kim K H, Payne D A, and Zuo J M (2012) Symmetry of piezoelectric (1-x) $\mathrm{Pb}\left(\mathrm{Mg}_{1 / 3} \mathrm{Nb}_{2 / 3}\right) \mathrm{O}_{3}-\mathrm{xpbTiO}_{3}(\mathrm{x}=0.31)$ single crystal at different length scales in the morphotropic phase boundary region. Phys. Rev. B 86, 184113.

Kim K H, Payne D A, and Zuo J M (2013b) Determination of fluctuations in local symmetry and measurement by convergent beam electron diffraction: applications to a relaxor-based ferroelectric crystal after thermal annealing. J. Appl. Cryst. 46, 1331-1337.

Kim K H, Xing H, Zuo J M, Zhang P, and Wang H (2015) TEM based high resolution and low-dose scanning electron nanodiffraction technique for nanostructure imaging and analysis. Micron 71, 39-45.

Kim K H and Zuo J M (2013) Symmetry quantification and mapping using convergent beam electron diffraction. Ultramicroscopy 124, 71-76.

Reetz M T, Maase M, Schilling T, and Tesche B (2000) Computer image processing of transmission electron micrograph pictures as a fast and reliable tool to analyze the size of nanoparticles. J. Phys. Chem. B 104, 8779-8781.

Zuo J M, Vartanyants I, Gao M, Zhang R, and Nagahara L A (2003) Atomic resolution imaging of a carbon nanotube from diffraction intensities. Science 300, 1419-1421. 


\section{Appendix. Script source for the geometric phase analysis}

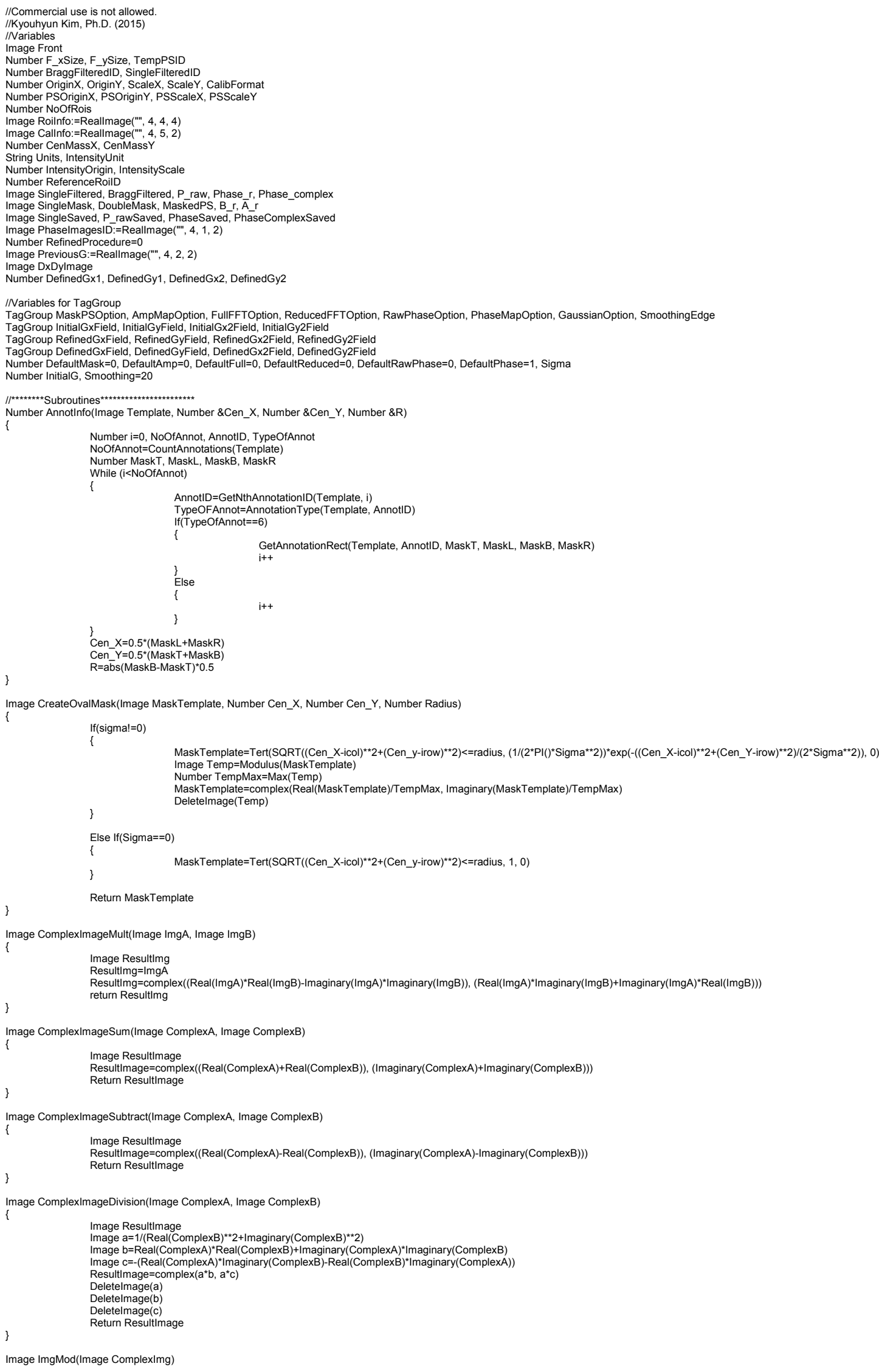




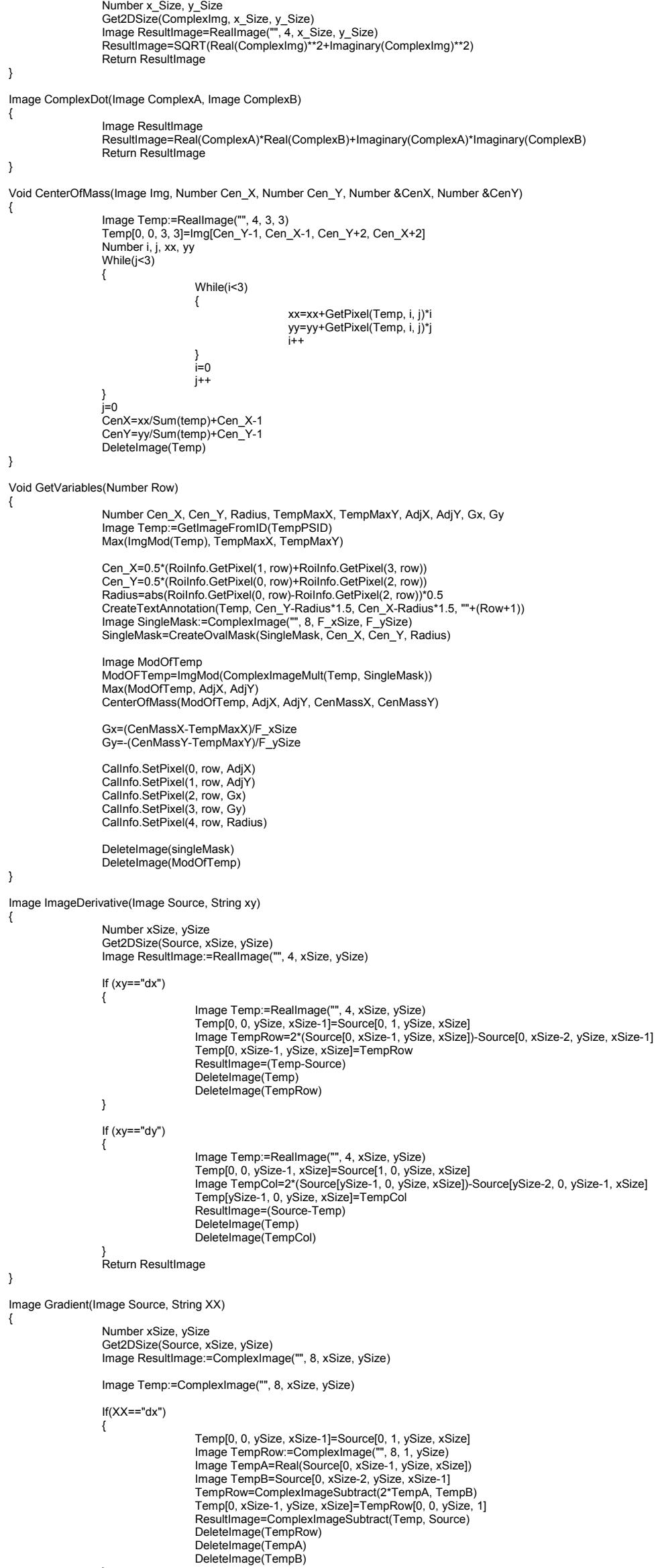




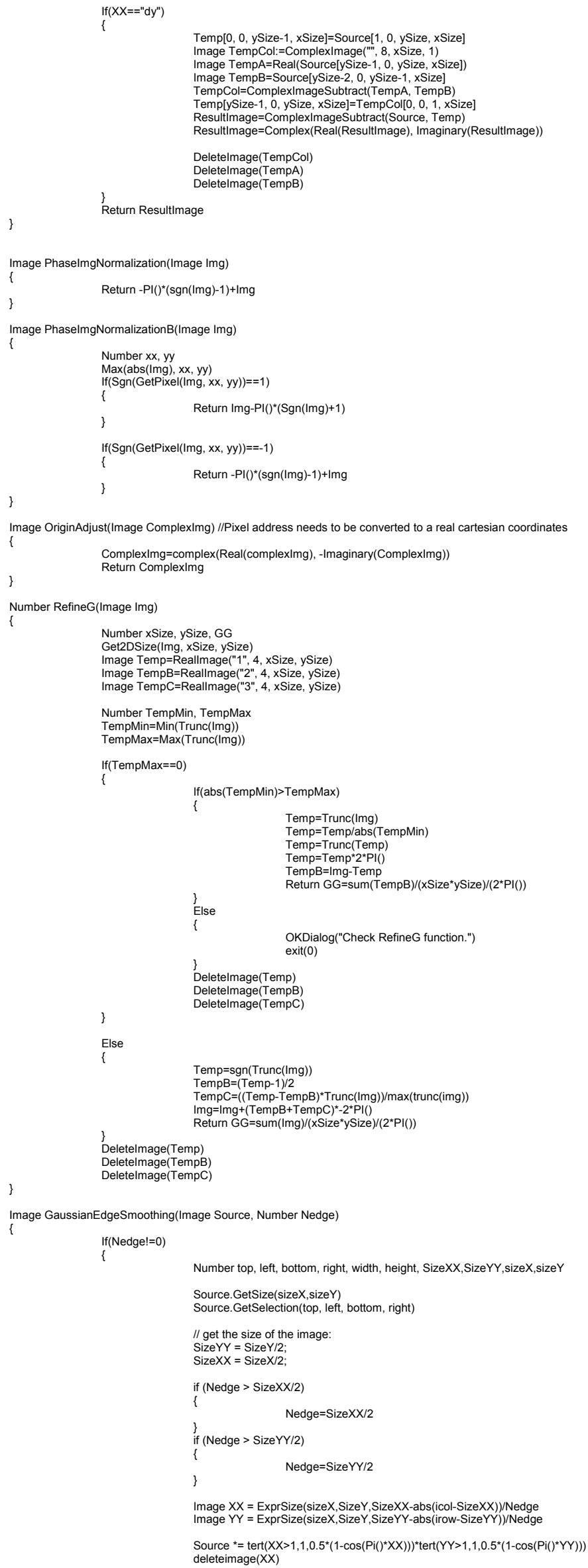




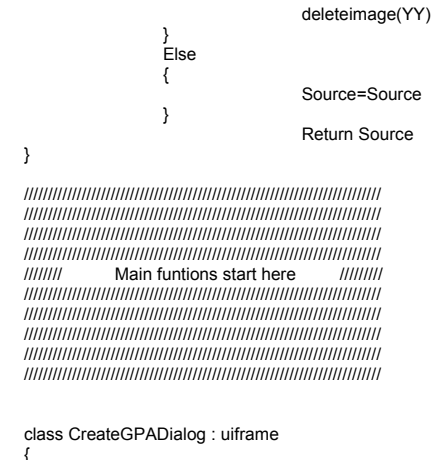

void M_FFT(object self)

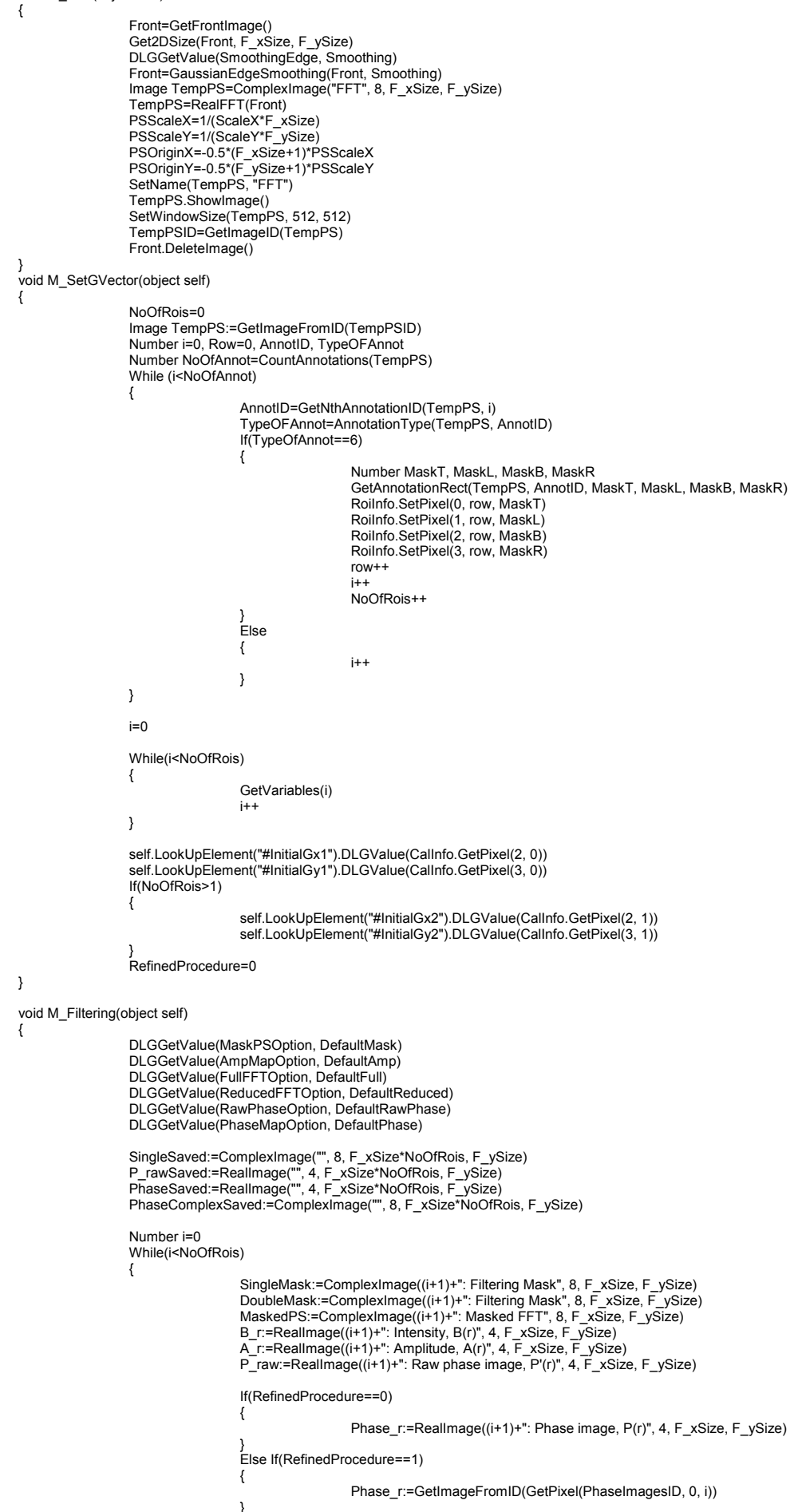


BraggFiltered:=Complexlmage((i+1)+": Bragg filtered for +-g, H(r)", 8, F_xSize, F_ySize) //H(r) SingleFiltered:=ComplexImage ((i+1)+": Bragg filtered for g, $H^{\prime}(r)^{\prime \prime}, 8, F_{-} x$ Size, F_ySize) $/ / H^{\prime}(r)$

Image TempPS:=GetlmageFromID(TempPSID)

MaskedPS=TempPS

SingleMask=CreateOvalMask(SingleMask, Callnfo.GetPixel(0, i), Callnfo.GetPixel(1, i), Callnfo.GetPixel(4, i))

DoubleMask=ComplexlmageSum(SingleMask, Rotate(SingleMask, Pi()))

//Bragg filtered for +-g, $\mathrm{H}(\mathrm{r})$

BraggFiltered=OriginAdjust(BraggFiltered)

//Bragg filtered for $\mathrm{g}, \mathrm{H}^{\prime}(\mathrm{r})$

SingleFiltered=IFFT(ComplexImageMult(MaskedPS, SingleMask))

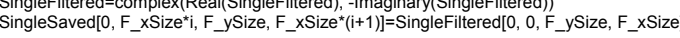

I/Intensity, $\mathrm{B}(\mathrm{r})$

B $r=2^{*}$ Real(SingleFiltered)

//Amplitude, $\mathrm{A}(\mathrm{r})$

A_ $r=\operatorname{lmgMod}($ SingleFiltered)

//Raw phase image, $\mathrm{P}^{\prime}(\mathrm{r})$

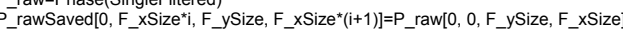

/IPhase image, $\mathrm{P}(\mathrm{r})$

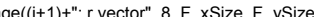

r. vector=complex(iCol, -iRow)

g_Vectorlmg=complex(Callnfo.GetPixel(2, i), Callnfo.GetPixel(3, i))

Image GdotR:=ComplexImage((i+1)+": GdotR", 8, F_xSize, F_ySize

GdotR $=\exp \left(2^{*} \mathrm{PI}()^{*} \mathrm{ComplexDot}\left(r_{-}\right.\right.$vector, g_vectorlmg)

ComplexDot( $($ vector, $g$ vectorlmg

Phase image, $P(r)^{n}, 8, F x$ Size, $F$ ySize

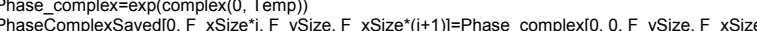

(

PhaseSaved[0, F_xSize $e^{\star} i, F_{-}$ySize, F_xSize $\left.{ }^{*}(i+1)\right]=P h a s e \_r\left[0,0, F_{-}\right.$ySize, F_xSize $]$

If(DefaultMask $==1)$

Image DisplayMaskedPS:=ComplexImage((i+1)+": Masked PS", 8, F xSize, F ySize $)$ If(DefaultFull $=0$ ) Else

DisplayMaskedPS=ComplexlmageMult(MaskedPS, SingleMask

DisplayMaskedPS=ComplexlmageMult(MaskedPS, DoubleMask)

Else If(DefaultMask $=0$

SetWindowSize(DisplayMaskedPS, 256, 256)

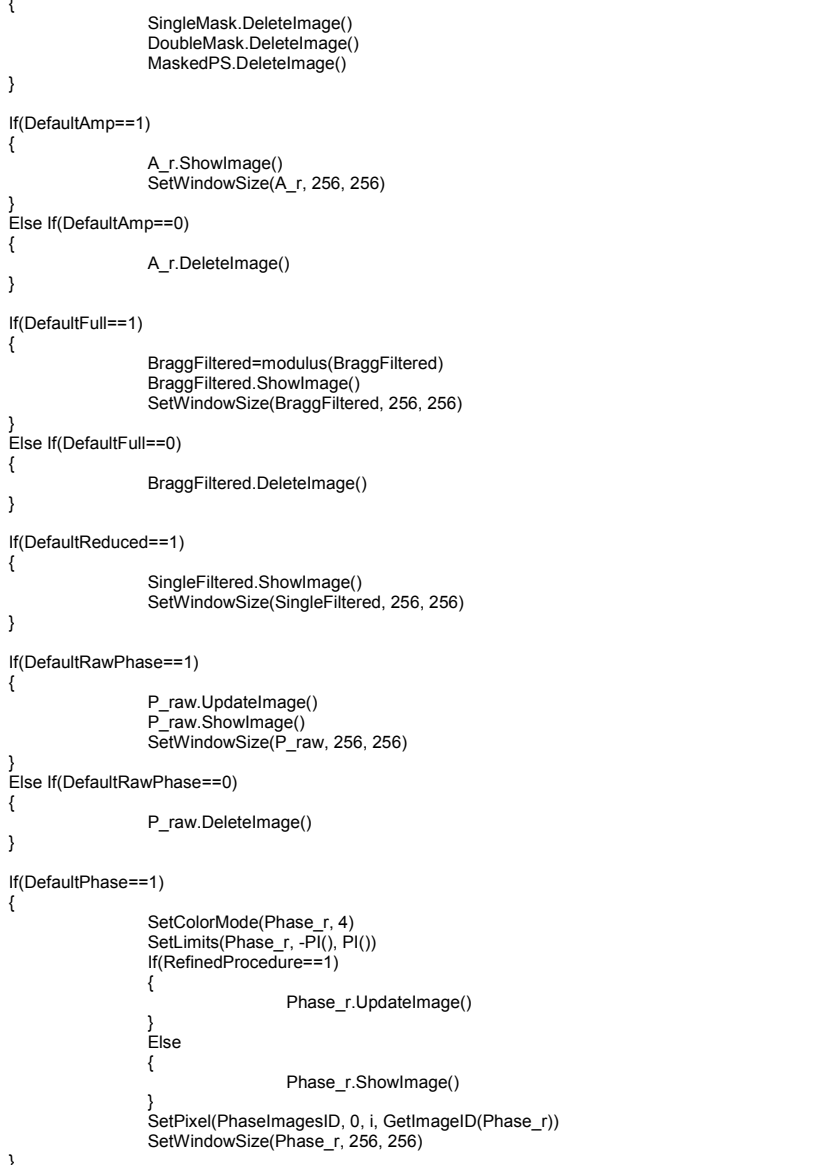




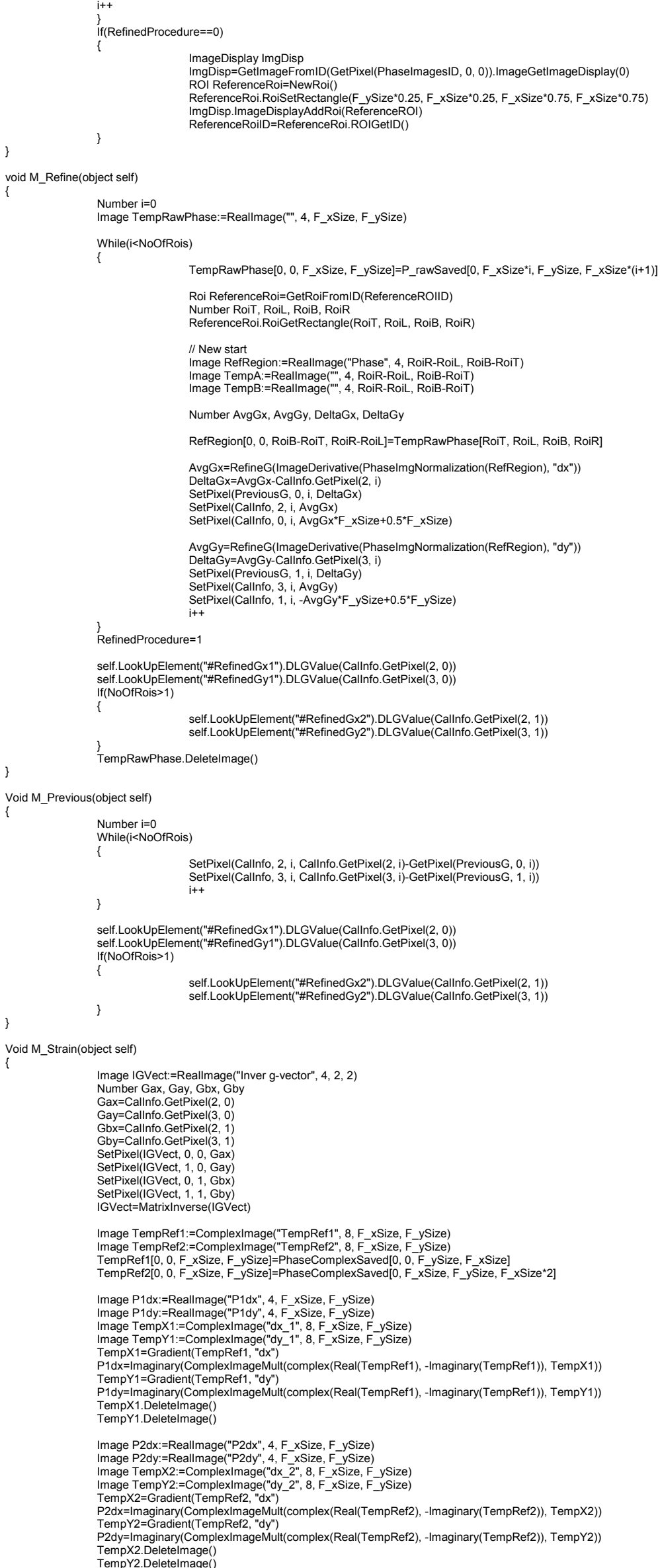




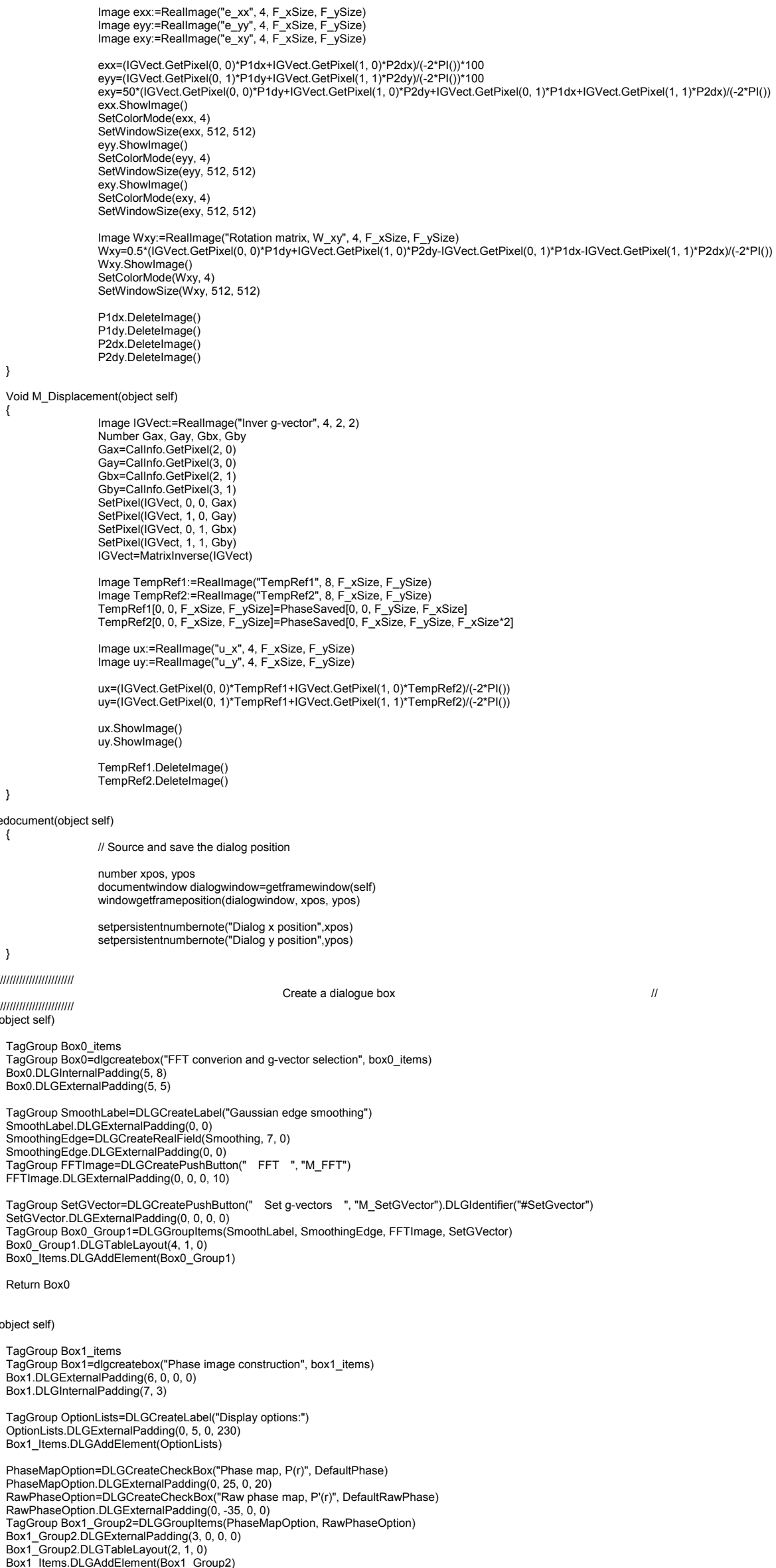

TagGroup Box0=dlgcreatebox("FFT converion and g-vector selection", box0_items) Box0.DLGInternalPadding $(5,8)$
Box0.DLGExternalPadding $(5,5)$

TagGroup SmoothLabel=DLGCreateLabel("Gaussian edge smoothing")

SmoothLabel.DLGExternalPadding $(0,0)$

SmoothingEdge.DLGExternalPadding(0, 0)

TagGroup FFTImage $=$ DLGCreatePushButton
FFTImage.DLGExternalPadding $(0,0,0,10)$

TagGroup SetGVector=DLGCreatePushButton(" Set g-vectors ", "M_SetGVector").DLGIdentifier("\#SetGvector")

SetGVector.DLGExternalPadding $(0,0,0,0)$

TagGroup Box0_Group1=DLGGroupltems(SmoothLabel, SmoothingEdge, FFTImage, SetGVector)

Box0_Items.DLGAddElement(Box0 1,0$)$

Return Box0

TagGroup Box1_items

mage construction", box 1 items)

Box1.DLGExternalPadding $(6,0,0,0)$
Box1.DLGInternalPadding $(7,3)$

TagGroup OptionLists=DLGCreateLabel("Display options:")

OptionLists.DLGExternalPadding $(0,5,0,230)$

PhaseMapOption=DLGCreateCheckBox("Phase map, P(r)", DefaultPhase)

PhaseMapOption.DLGExternalPadding $(0,25,0,20)$

RawPhaseOption=DLGCreateCheckBox("Raw phase map, $P^{\prime}(r)$ ", DefaultRawPhase)

RawPhaseOption.DLGExternalPadding $(0,-35,0,0)$

TagGroup Box1_Group2=DLGGroupltems(PhaseMapOption, RawPhaseOption)

Box1_Group2.DLGExternalPadding $(3,0,0,0)$

Box1_tems.DAddElement(Box1

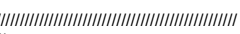

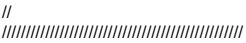
TagGroup BoxO(object self)

TagGroup Box1(object self) 


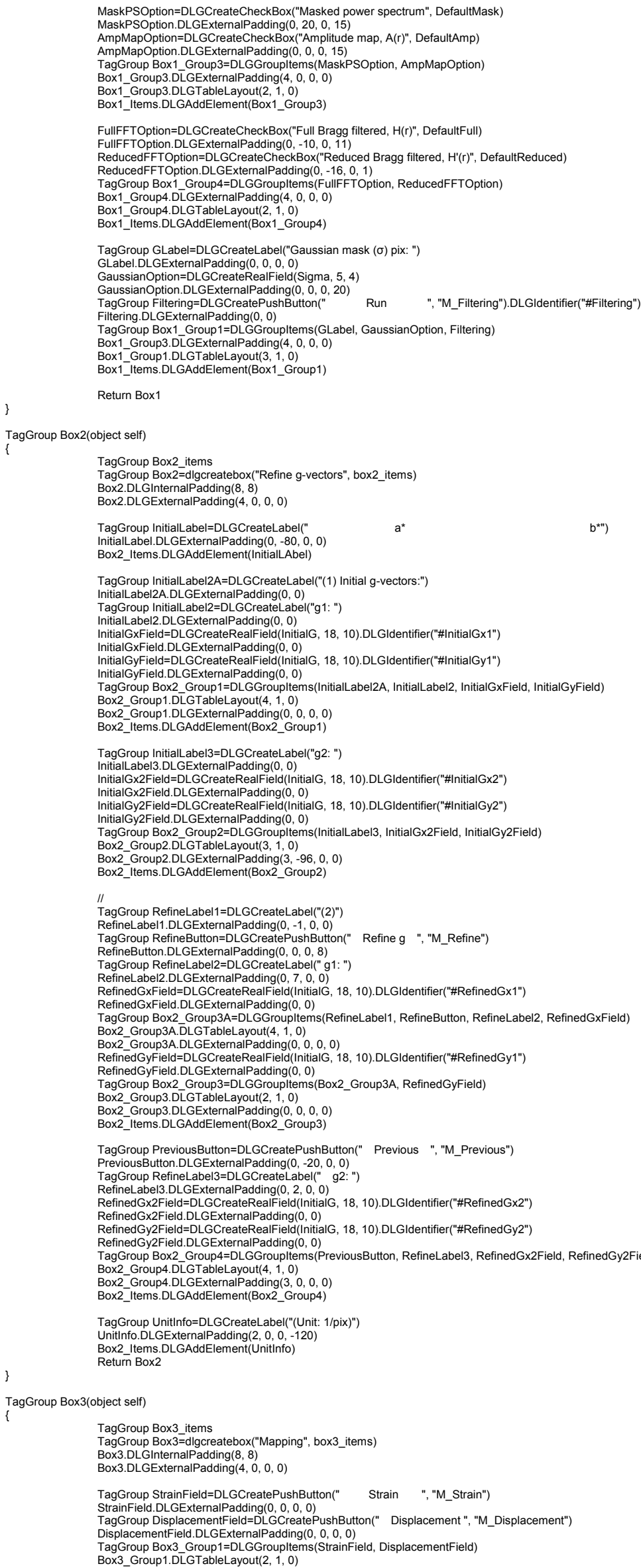


Box3_ttems.DLGAddElement(Box3_Group1)

Return Box3

(

taggroup Mylnfo(object self)

Taggroup Mylnfo=dlgcreatelabel("Kyouhyun Kim, Ph.D., 2015, All rights reservedln") Mylnfo.dlgexternalpadding $(0,0)$ return Mylnfo

TagGroup CreateDialog(object self)

TagGroup dialog items;

TagGroup dialog = DLGCreateDialog("GPA analysis", dialog_items)

// Call each buttons

Dialog_items.DLGAddElement(Self.Box0());

Dialog_items.DLGAddElement(Self.Box1())

Dialog_items.DLGAddElement(Self.Box2();

Dialog_items.DLGAddElement(Self.Mylnfo())

Return Dialog

CreateGPADialog(object self)

Self.Super.Init(Self.CreateDialog())

number xpos, ypos

getpersistentnumbernote("Dialog y position",ypos)

self.display("Geometric Phase Analysis Ver 1.0")

documentwindow dialogwin=getdocumentwindow $(0)$

if(xpos! $=0$ \&\& ypos! $=0)$

windowsetframeposition(dialogwin, xpos, ypos)

C CreateGPADialog(object self)

Alloc(CreateGPADialog) 